\title{
Momentum distribution of multiply charged ions produced by intense $\left(50-70-\mathrm{PW} / \mathrm{cm}^{2}\right)$ lasers
}

\author{
K. I. Dimitriou, ${ }^{1,2}$ S. Yoshida, ${ }^{1}$ J. Burgdörfer, ${ }^{1,4}$ H. Shimada,${ }^{3,4}$ H. Oyama, ${ }^{4}$ and Y. Yamazaki ${ }^{3,4}$ \\ ${ }^{1}$ Institute for Theoretical Physics, Vienna University of Technology, A-1040 Vienna, Austria, EU \\ ${ }^{2}$ Physics Department, National Technical University, Athens, Greece, EU \\ and Theoretical and Physical Chemistry Institute, National Hellenic Research Foundation, Athens 11635, Greece, EU \\ ${ }^{3}$ Graduate School of Arts and Sciences, University of Tokyo, Toyko 153-8902, Japan \\ ${ }^{4}$ Atomic Physics Laboratory, RIKEN, Saitama 351-0198, Japan
}

(Received 24 May 2006; published 24 January 2007)

\begin{abstract}
We investigate both theoretically and experimentally the momentum distribution of multiply charged ions ionized by an intense multicycle laser field with a maximum intensity of $\sim(50-70) \mathrm{PW} / \mathrm{cm}^{2}$. Ions with different charge states are produced during a single laser shot due to a spatial variation of the laser intensity within the beam focus. The measurements show approximately a simple linear relation between the width of the momentum distributions and the ionization potential of the ions. Such a power law scaling appears to be universal for various rare gas atoms used ( $\mathrm{He}, \mathrm{Ne}, \mathrm{Ar})$. We analyze this ionization dynamics using a quasiclassical tunneling theory for a single active electron model assuming that the interaction between electrons is negligible in such a strong field limit. We show that for the relatively long pulses used in the present work ( $\sim 200$ fs or $\sim 80$ cycles) the effect of the laser envelope plays an important role in the ionization process.
\end{abstract}

DOI: 10.1103/PhysRevA.75.013418

PACS number(s): 32.80.Rm, 32.80.Fb, 03.65.Sq

\section{INTRODUCTION}

During the last years, single and double ionization of neutral atoms by infrared laser pulses has been intensively studied both experimentally [1-3] and theoretically [4-6]. These studies, particularly for double ionization, are focused on the calculation of the ion yields and reveal two basic mechanisms for the production of the same charge state: Sequential ionization is observed at high fields with two electrons being ionized independently of each of them and successively removed. Nonsequential ionization is observed at low fields where the electron ionized first will knock out the second (bound) electron in a laser assisted electron-electron collision, leading to double ionization. Very recent measurements [7] of ion yields revealed that these two mechanisms are also present for multiple ionization involving more electrons (up to $\mathrm{Ne}^{5+}$ ). In the strong-field regime the measured yields agree with the Ammosov, Delone, and Krainov tunneling (ADK) theory $[8,9]$ developed within a single active electron model, which implies that electrons in the strong-field regime are independent and therefore the ionization is sequential. The sequential mechanism in multiple ionization of atoms irradiated by a vuv laser pulse has also been confirmed in recent experiments [10].

Technological advances in laser and in atomic collision physics have opened new pathways for measurements beyond the ion yield production. Foremost, the momentum distribution of ionized atoms produced by linearly polarized lasers with relatively moderate intensity has been intensively analyzed employing cold-target recoil-ion-momentum spectroscopy [11-18]. The measured distributions of the doubly charged ion momentum parallel to the polarization of the laser show for $\mathrm{Ne}$ and Ar two symmetrical peaks with a dip at zero momentum. This is an indication of the strong electron-electron interaction in the system where the ion gains high recoil momentum from ionized electron by rescattering $[19,20]$. In the intense field regime, highly charged ions are produced by intense laser fields and the momentum distributions of each individual ion show Gaussian-like distributions centered at zero momentum [13,14,21,22]. This suggests that the ionization dynamics is mainly dominated by a sequential process and consequently the electronelectron interaction is relatively weak.

In this paper we explore the regime with a very intense laser field by which up to an eight fold charged ion can be produced. The driving field used in the measurements is a linearly polarized laser field approximated by

$$
F(t)=F_{\max } f(t) \sin (\omega t+\phi) \quad(0<t<\tau),
$$

where $F_{\max }$ is a peak field strength, $\omega$ a laser frequency, $\phi$ the carrier envelope phase, and $f(t)=\sin ^{2}(\pi t / \tau)$ the approximate laser envelope. Since we study in the present paper relatively long pulses with full width at half maximum (FWHM) of about 200 fs or about 80 optical cycles, details of the carrier envelope phase are unlikely to be important. Due to the focusing of the laser, $F_{\max }$ is not spatially homogeneous in the interaction region, i.e., the peak intensity $I_{\max }=(c / 8 \pi) F_{\max }^{2}$ has a maximum at the focal point and decreases with increasing distance from the focal point. (Atomic units are used throughout unless otherwise stated.) Since the target gas beam has a spatial spread, atoms experience different intensities depending on their position relative to the focal point of the laser. The higher the intensity, the more electrons are ionized. As a consequence of the spatial spread, a broad charge state distribution is produced within a single laser shot. The longitudinal momentum distribution of such ions with individual charged state is analyzed. We will focus on the width of the momentum distributions and show that the width displays a power-law behavior as a function of the ionization potential of each ion with an exponent close to unity. This dependence is found for the width for different target atoms. 
The theoretical investigation of multiple ionization is not easy to implement. As the number of electrons involved increases, the $a b$ initio calculation of the many-body dynamics becomes beyond the reach of the currently available computing power. To attack such a complicated system, therefore, a drastic simplification is inevitable. Judging from the studies of ionization rates and momentum distributions mentioned above, the laser intensity used in this study is so strong that only the sequential ionization can be expected to dominate. For the theoretical study of the momentum distribution of multiply charged ions we, therefore, employ a single active electron model. A comparison with measured results suggests the validity of this simplified model for the complicated multiple ionization dynamics. We simulate the dynamics using the classical trajectory Monte Carlo method including tunneling (CTMC-T method [23]). For a very intense laser it is commonly assumed that electrons ionize over the barrier rather than by tunneling. However, when the pulse duration is long, tunneling plays a significant role in determining the final state of electrons and ions. Electrons ionize long before a laser field reaches the peak intensity. The final state of an electron is determined by the laser intensity at the time of tunneling [24] and not by the peak intensity. Therefore the effect of tunneling and the temporal field strength during tunneling plays a key role for this multiple ionization process. In the following sections we introduce the experimental setup used in this investigation, and then the theoretical model. We analyze the results by comparing the measured data with simulations.

\section{EXPERIMENTAL SETUP}

The laser system is based on the chirped pulse amplification (CPA) technique [25]. A stretched light pulse $(\sim 1 \mathrm{~mJ}$, 775 nm, i.e., $\omega=0.059)$ from a seed laser system (CPA 2001, Clark-MXR) is amplified up to $\sim 300 \mathrm{~mJ}$ by a Ti:S four-pass amplifier pumped with a $\sim 1.5 \mathrm{~J}$ pulse from a frequencydoubled Nd:YAG laser (Continuum) operated at $10 \mathrm{~Hz}$. The amplified light pulse is compressed by a pair of parallel gratings. After compression, the duration of the light pulse is $\sim 200 \mathrm{fs}$ (FWHM) and the energy is decreased to $\sim 120 \mathrm{~mJ}$.

The light is sent to the vacuum chamber through a fusedsilica window. The residual gas pressure of the chamber is lower than $4 \times 10^{-10}$ Torr. In the vacuum chamber, the light is focused onto an effusive rare gas beam ( $\mathrm{Ne}, \mathrm{Ar}, \mathrm{Kr}$ ) with an off-axis parabolic mirror with a focal length of $300 \mathrm{~mm}$. The laser beam profile at the focal plane is nearly circular with a radius, $R_{0}$, of $\sim 15 \mu \mathrm{m}$ at half maximum. The spatial dependence of the peak intensity near the focal plane can be approximately represented as

$$
I_{\max }(X, Y, Z)=I_{\text {focal }} \frac{R_{0}^{2}}{\bar{R}(X)^{2}} \exp \left(-\frac{Y^{2}+Z^{2}}{2 \bar{R}(X)^{2}}\right),
$$

where $I_{\text {focal }}$ is the maximum intensity estimated to be $\sim(5$ $-7) \times 10^{16} \mathrm{~W} / \mathrm{cm}^{2}$ and $\bar{R}(X)$ is the radius of laser beam at the plane $x=X$ perpendicular to the propagation direction, i.e., $\bar{R}(0)=R_{0}$.

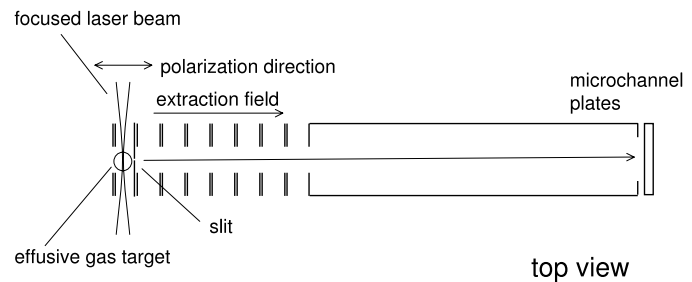

FIG. 1. A schematic drawing of the time-of-flight analyzer.

The laser beam is crossed with a collimated effusive beam (diameter: $D_{A}=3 \mathrm{~mm}$ ) of the gas target [Fig. 1]. While the target beam has a thermal $(300 \mathrm{~K})$ momentum spread along its direction, the momentum spread along the light polarization is small compared with the momentum acquired in the course of ionization. This momentum spread is estimated from the distance between the nozzle and the crossing point $(60 \mathrm{~mm})$, and the extent of the reaction volume $(30 \mu \mathrm{m})$. This thermal contribution is about $6 \%$ of the experimentally measured width for the case of $\mathrm{Kr}^{+}$and smaller for larger charge states or larger ionization potential. The density of the gas target is $\sim 10^{6} \mathrm{~cm}^{-3}$, which is about one order of magnitude lower than the residual gas. This low target density is adopted to assure that the observed momentum distribution is not distorted by space charge effects and multiple hits of target ions of the same charge state on the multichannel plate (MCP). In the region distant from the focal plane, large numbers of low charge state ions are produced since the peak intensity is low. The number of the detected ions per laser shot is less than 0.2 for each charge state. In order to detect only ions produced near the focal plane, a slit with a width of $d_{s}=0.5 \mathrm{~mm}$ is placed $5 \mathrm{~mm}$ downstream from the target.

The charge state of the ions as well as the momentum component parallel to the light polarization are measured using the time-of-flight (TOF) technique. Multiply charged ions produced by the focused laser light are extracted by a uniform static electric field of about $27 \mathrm{~V} \mathrm{~cm}^{-1}$. The direction of the electric field is set parallel to the light polarization. The extraction region $(75 \mathrm{~mm})$ is followed by a drift region $(150 \mathrm{~mm})$, and the extracted ions are detected by a stack of multichannel plates placed on the other side of the tube. The time-of-flight of the ion is recorded by a multipleevent time digitizer (P7886, FAST ComTec $\mathrm{GmbH}$ ) with a time resolution of $0.5 \mathrm{~ns}$. A typical width of the peak of the TOF spectrum is $\sim 7 \mathrm{~ns}$. Because the pulse-pair resolution of the whole detection system is limited to about 5 ns (the main contribution comes from the pulse width of MCPs), the detectable number of ions per laser shot is, in most cases, one for each charge state. Assuming a Poisson distribution for the number of produced ions per laser shot and using the value of 0.2 as an upper limit for the number of the detected ions, a probability that more than one ion is produced can be estimated to be less than $3 \%$. The resulting momentum spectra are compared using theoretical models discussed in the following sections.

\section{INDEPENDENT ELECTRON MODEL}

For the production of the ion with a certain charge state $\mathrm{A}^{q+}$, we consider a system with $q$ active electrons described by the Hamiltonian 


$$
H_{q}=\sum_{i=1}^{q}\left(\frac{p_{i}^{2}}{2}+V_{q}\left(r_{i}\right)+z_{i} F(t)\right)+V_{\mathrm{int}}\left(\vec{r}_{1}, \ldots, \vec{r}_{q}\right) .
$$

$V_{\text {int }}$ is the interaction potential among $q$ electrons to be detached, each of which interacts with the nonactive electrons through $V_{q}\left(r_{i}\right)$ representing the core potential of the $\mathrm{A}^{q+}$ ion screened by those passive electrons. For example, to calculate the $\mathrm{Ne}^{q+}$ ion yield the model potential corresponds to the ion core $-10 / r$ screened by $10-q$ electrons, i.e., $V_{q}(r \rightarrow 0)$ $=-10 / r$ and $V_{q}(r \rightarrow \infty)=-q / r$. The number of active electrons $q$ is determined by the laser intensity relative to the $q$ th ionization potential of the atom (discussed more in detail later). Since the simulation of the $q$-electron system is quite complicated, we simplify the model drastically. For high laser intensity and long laser duration used in this study, sequential ionization is expected to dominate. Correspondingly, recollisions or, more generally, electron-electron correlation is assumed to be small. Thus we neglect the interaction term $V_{\text {int }}$ in Eq. (3). Consequently the dynamics of electrons can be simplified due to the separability of the Hamiltonian in the absence of $V_{\text {int }}$. Each electron follows the equations of motion by the single active electron model associated with the Hamiltonian

$$
H_{1}=\frac{p^{2}}{2}+V_{q}(r)+z F(t)
$$

In the CTMC-T code we use for $V_{q}(r)$ the model potentials proposed by $[26,27]$ which describe the interaction of a single electron with the ion core of charge state $A^{q+}$ screened by nonactive electrons. As long as the interactions between active electrons can be neglected in comparison with the laser interaction term $z F(t)$, the dynamics of electrons can be approximated by an independent electron model.

As mentioned above, the peak intensity $I_{\max }$ (or equivalently the peak field $F_{\max }$ ) determines the number of the emitted (active) electrons $q$. We will deduce now the fraction of atoms in the interaction region experiencing a certain peak intensity due to the spatial intensity variation of the laser [Eq. (2)] or, in other words, in which interaction region $(X, Y, Z)$, ions of a given charge state $A^{q+}$ are produced. The peak intensity $I_{\max }$ varies with the position of atoms relative to the focal point of the laser, $(X, Y, Z)$. We note that the motion of the parent atom is neglected and therefore $I_{\max }$, the peak of envelope, is a constant for each individual atom during the interaction with laser. Since the $A^{q+}$ ion yield is observed when the intensity exceeds a certain intensity $I_{q}$ which is called the appearance intensity for $A^{q+}$ ions, the parent atom $A$ is confined inside the volume $Y^{2}+Z^{2}$ $<R\left(I_{q}, X\right)^{2}$ where

$$
R\left(I_{q}, X\right)^{2}=-2 \bar{R}(X)^{2} \ln \left(\frac{I_{q}}{I_{\text {focal }}} \frac{\bar{R}(X)^{2}}{\bar{R}(0)^{2}}\right),
$$

with $R^{2}=Y^{2}+Z^{2}$ which follows from Eq. (2). For stronger fields an additional (or even several) electron(s) is (are) detached and yields $A(q+1)+$ ions. The $A^{q+}$ yield results therefore from the region $R\left(I_{q+1}, X\right)^{2}<Y^{2}+Z^{2}<R\left(I_{q}, X\right)^{2}$ (Fig. 2). Equivalently, the atoms exposed to the laser field with a peak

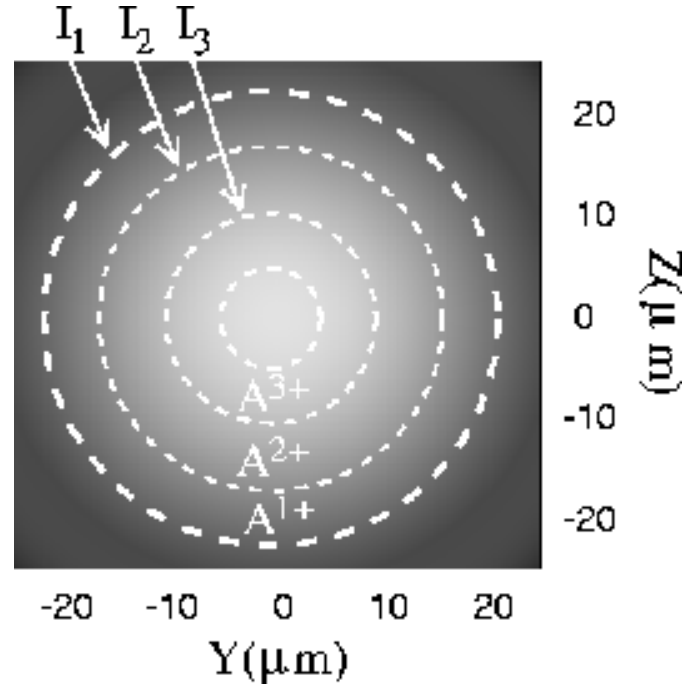

FIG. 2. Cross section of the laser beam in the focal plane (schematic picture). Intensity distribution is depicted as colored contour plot in log scale. The region where the corresponding ions are produced is indicated.

intensity in the interval $I_{q}<I_{\max }<I_{q+1}$ result in ions of charge state $q^{+}$. The density $n_{a}$ of atoms is approximately constant inside the interaction region since the laser beam radius and the slit size is much smaller than the atomic beam radius, i.e., $D_{A} \gg \bar{R}(X), d_{s}$. Thus the number of atoms

$$
N(X) \Delta I=2 \pi n_{a} R\left(I_{\max }, X\right)\left|\frac{d R}{d I}\right| \Delta I=2 \pi n_{a} \frac{\bar{R}(X)^{2}}{I_{\max }} \Delta I
$$

is exposed to a peak intensity of the laser within the interval $\left[I_{\max }, I_{\max }+\Delta I\right]$ in a fixed plane at $x=X$. Therefore the integration over the interaction region

$$
\rho\left(I_{\max }\right)=C \int_{-d_{s} / 2}^{d_{s} / 2} N(X) d X \propto \frac{1}{I_{\max }}
$$

gives the total intensity distribution that the initially neutral atoms experience [28]. Here $C$ is the normalization constant. For a simplicity we will use the appearance intensity $I_{q}$ for the $A^{q+}$ ion determined by the classical threshold, i.e.,

$$
I_{q}=\frac{c}{8 \pi} \frac{E_{\mathrm{ion}}(q)^{4}}{16 q^{2}},
$$

where $E_{\text {ion }}(q)$ is the $q$ th ionization potential of the atom $A$ [29]. The sensitivity of the calculated momentum distribution on this choice [Eq. (8)] is, however, very small, as will be discussed later.

\section{CTMC-T METHOD}

The dynamics of the system which is reduced to a single active electron model [Eq. (4)] is simulated by the CTMC-T method $[23,30]$ which gives us the advantage that it can be easily implemented to average over different peak intensities following the distribution shown above [Eq. (2)]. Initially, an ensemble of electrons each of which belongs to a different 
ion $A^{(q-1)+}$ is generated according to the microcanonical ensemble [31,32] for a screened ion core $V_{q}(r)$ with an ionization potential $E_{\text {ion }}(q)$. Each electron follows the equations of motion with the Hamiltonian [Eq. (4)]. In order to investigate the momentum distribution of the $A^{q+}$ ion under the present experimental conditions, we generate for each individual electron trajectory a uniformly distributed random carrier envelope phase distribution and a random peak intensity weighted by the distribution [Eq. (2)] within a range of $I_{q}$ $<I_{\max }<I_{q+1}$ as discussed above. Every time the electron reaches a turning point, i.e., $p_{z}=0$ and $z F(t)<0$, the WKB tunneling probability $w_{\mathrm{tnn}}$ is calculated (for details see $[23,30])$. The trajectory bifurcates at this moment: The trajectory either tunnels through the barrier with the probability for tunneling $W_{\text {tot }}=1-\left(1-w_{\text {tnn }}\right)^{\alpha(q)}$ or continues on its trajectory inside the barrier with $1-W_{\text {tot }}=\left(1-w_{\text {tnn }}\right)^{\alpha(q)}$. The probability $W_{\text {tot }}$ represents a time-integral over the tunneling rate

$$
\frac{d W_{\mathrm{tot}}}{d t}=-\alpha(q) \frac{d w_{\mathrm{tnn}}}{d t}\left(1-w_{\mathrm{tnn}}\right)^{\alpha(q)-1},
$$

where $\alpha(q)$ is the number of equivalent electrons in the atom. Equation (9) is of binomial form in line with the independent electron approximation. It is simply a product of the tunneling rate of a single electron, of $d w_{\mathrm{tnn}} / d t$, and of the probability that $\alpha(q)-1$ electrons remain inside the barrier multiplied by the number of possible permutations, $\alpha(q)$. In the simulation we choose $\alpha(q)=3-q(q=1,2)$ for atoms with the ground state electron in an $s$-state and $\alpha(q)=7-q(q$ $=1,2, \ldots, 6)$ in a $p$-state. This method to treat $\alpha(q)$ equivalent electrons is similar to the $n$-CTMC method developed for the collision dynamics [33]. This bifurcation procedure is repeated along the electron trajectory until the end of the pulse is reached. For example, the trajectory which tunnels at the next turning point with a probability $W_{\text {tot }}^{\prime}$ is weighted by $\left(1-W_{\text {tot }}\right) W_{\text {tot }}^{\prime}$, The total statistical weight summed over all the bifurcated trajectories originating from a single set of initial conditions of the microcanonical ensemble adds up to one. Therefore the contribution of trajectories tunneled at different times and, moreover, the depletion of the parent state are properly and effectively accounted for during the time evolution. We note that a minimum tunneling probability is typically set to $10^{-4}$ (this number is varied with the intensity). Below the minimum value no tunneling is included in order to avoid the enormously large number of (ineffective) bifurcations. After the conclusion of a pulse the final state of each trajectory is calculated analytically at $t$ $=\infty$. The distribution of a momentum component parallel to the laser polarization can be thus obtained from the ensemble of trajectories. The resulting distribution and its width will be compared with the measurements in the next section.

\section{LONGITUDINAL MOMENTUM DISTRIBUTION}

Figure 3 shows a longitudinal momentum distributions of $\mathrm{Ne}^{+}$driven by a laser pulse with a 200 fs (FWHM) pulse duration, a wavelength of $775 \mathrm{~nm}(\omega=0.059$ a.u. $)$, and a peak intensity of $50-70 \mathrm{PW} / \mathrm{cm}^{2}$. The measured spectrum is well-reproduced by the simulation. For reference, we also

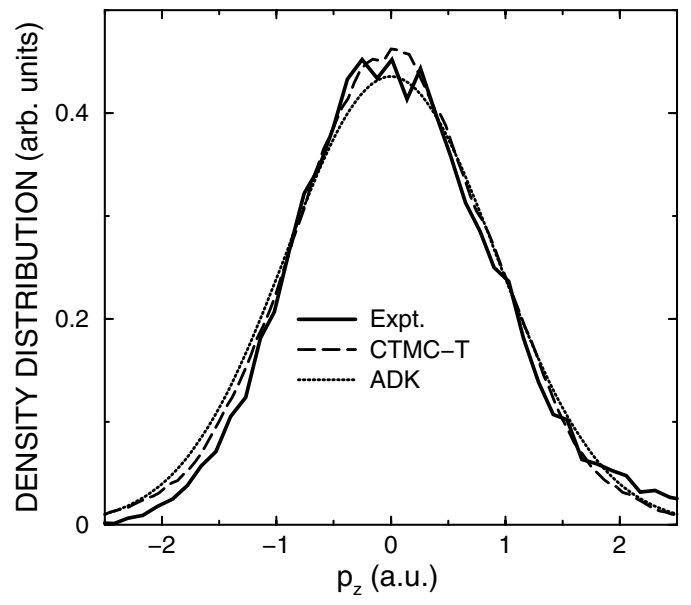

FIG. 3. Longitudinal momentum distributions of $\mathrm{Ne}^{+}$. Solid line: measured distribution, dashed line: CTMC-T, and dotted line: ADK. For the CTMC, the number of equivalent electrons is set to $\alpha(1)=6$.

plot the distribution predicted by the ADK theory $[8,9]$. Due to the very intense laser field, the Gaussian-like distribution, i.e.,

$$
g\left(p_{z}\right) \propto \exp \left(-\frac{p_{z}^{2}}{2\left[\delta p_{\mathrm{ADK}}(I)\right]^{2}}\right)
$$

where

$$
\delta p_{\mathrm{ADK}}(I)=\sqrt{\frac{3}{2 \omega^{2}}}\left(\frac{I}{2 E_{\mathrm{ion}}(q)}\right)^{3 / 4}
$$

(see Ref. [9]) is observed rather than a more complex structure seen for the lower laser intensity [17]. The width of the distribution from the ADK theory seems to agree with the measured spectrum when the estimate is made using a fixed peak intensity $I=I_{1}$ which is the lower boundary of the intensity interval pertaining to the charge state $q=1$. A more detailed comparison between the measurements and the two different theories can be made for different target atoms and charge states (Fig. 4) where the momentum distribution widths are plotted as a function of ionization potential. We note that for the ADK estimate a factor of $2 \sqrt{2 \log 2}$ is included to compare with the measured data in FWHM. Interestingly, the measured width depends on the ionization potential as $\delta p \propto E_{\text {ion }}^{\gamma}(q)$ with $\gamma$ close to unity. This dependence is almost independent of the target atom (the $\chi^{2}$ deviation is, on average, $\chi^{2} \simeq 0.88$ ). The CTMC-T results approximately reproduce such a nearly linear power-law scaling in agreement with measured data. We also show the two limiting cases of a power-law behavior derived from the ADK theory. $\delta p_{\mathrm{ADK}} \propto E_{\mathrm{ion}}(q)^{9 / 4}$ for a same charge state and different target atoms and $\delta p_{\mathrm{ADK}} \propto E_{\text {ion }}(q)^{3 / 4}$ for a same target and different charge states assuming $E_{\text {ion }}(q) \propto q$ [see Eqs. (8) and (11)]. By fitting the data for different targets to a single power law in the ionization potential, i.e., $\Delta p \propto E_{\text {ion }}^{\gamma}$, the measured widths yield $\gamma=1.08$, the CTMC widths $\gamma=1.01$, and the ADK widths $\gamma=0.875$. The exponent of the power law can be determined with smaller $\chi^{2}$ when the fitting is done for differ- 


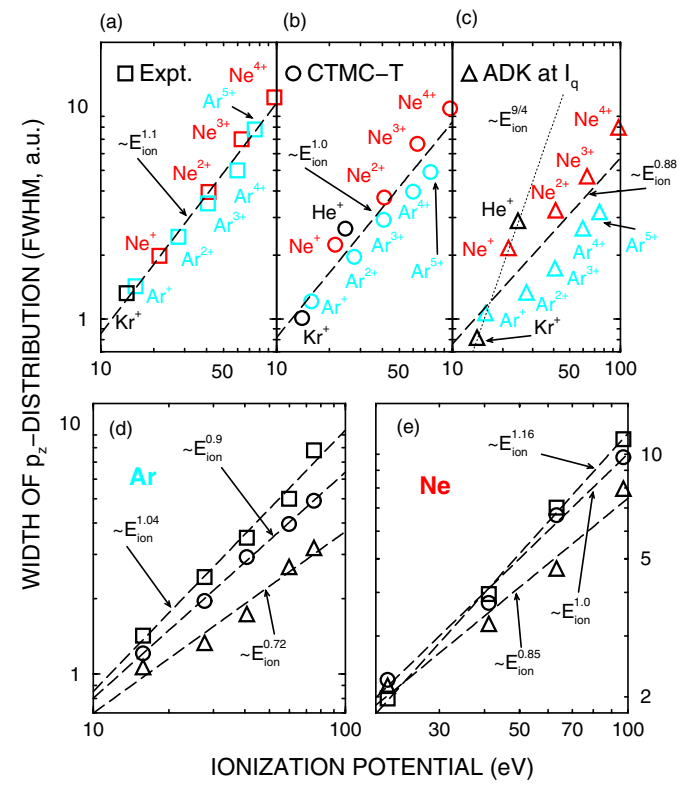

FIG. 4. (Color online) Width (FWHM) of the ion momentum distribution as a function of $E_{\text {ion }}(q)$. The atoms are subject to a $200 \mathrm{fs}$ laser pulse with a wavelength of $775 \mathrm{~nm}$ and a peak intensity of 50-70 $\mathrm{PW} / \mathrm{cm}^{2}$. (a) Measured results (squares), (b) the results simulated by the CTMC-T (circles), and (c) the ADK estimates (triangles) are plotted. In (d) and (e) the different atomic species are plotted separately and the definition of the symbols is the same as in (a)-(c). The results are fitted to a power law $\Delta p \propto E_{\text {ion }}^{\gamma}$ and depicted by the dashed lines. In (c) a power-law behavior $\Delta p \propto E_{\text {ion }}^{9 / 4}$ estimated by the ADK theory (see the text in detail) is also plotted (dotted line).

ent atomic species separately. For $\mathrm{Ne}$, we find $\gamma=1.16$ (expt.), 1.004 (CTMC), 0.853 (ADK) and for Ar $\gamma=1.04$ (expt.), 0.901 (CTMC), and 0.724 (ADK). Thus the CTMC results for the exponent $\gamma$ agree with the measured data within an error of $10 \%$ while the ADK underestimates the exponent by $20 \%-30 \%$. The largest absolute deviation from the experiment appears at the lowest ionization potential $\left[E_{\text {ion }}(q=1) \simeq 14 \mathrm{eV}\right]$ for krypton.

For an ionization potential near $21 \mathrm{eV}\left(\mathrm{Ne}^{+}\right)$the different models happen to coincide leading to the fortuitous agreement of Fig. 3. The good agreement seen in Fig. 3 thus seems to be coincidental. This is not surprising considering the fact that neither the effect of laser envelope nor the intensity averaging is taken into account in the ADK estimates. The standard ADK approximation has been developed for systems driven by a monochromatic laser field. As recently reported, a temporal laser profile at the momentum of ionization (or tunneling) determines the final state of ionized electrons, i.e., the final angular momentum [24] and the final energy [34]. Therefore in order to estimate a proper momentum width using the ADK theory, $\delta p_{\mathrm{ADK}}(I)$ has to be averaged over a temporal laser intensity at the moment of ionization rather than over a peak intensity [Eq. (7)]. Since the distribution of the temporal laser intensity effective for ionization is a convolution of a peak intensity distribution and a time dependent ionization rate determined by laser envelope, the averaging can be quite complicated. For a short and rela-

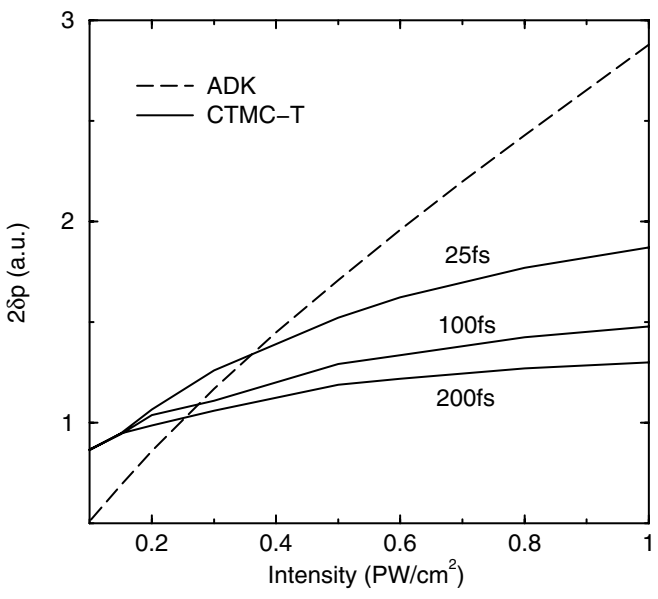

FIG. 5. Momentum distribution width of the $\mathrm{H}^{+}$ion driven by laser with a wavelength of $775 \mathrm{~nm}$ and pulse durations $(25,100$, and $200 \mathrm{fs}$ ). The width is plotted as a function of the peak intensity of the laser. (No intensity averaging is done.) In the ADK estimate the momentum width is evaluated using the peak intensity and independent of the pulse duration.

tively weak laser, ionization takes place within a few halfcycles of laser and this averaging does not play an important role. However, in the present case of a long $(\sim 200 \mathrm{fs})$ and intense laser pulse, ionization takes place over many halfcycles each of which has a different maximum amplitude. Therefore the averaging considerably affects the width of momentum distributions.

Figure 5 shows the width of the momentum distribution predicted by the ADK model [Eq. (11)] together with the width estimated by the CTMC-T simulation for a fixed peak intensity for hydrogen. A similar behavior is observed for rare gas atoms. Considering the fact that the CTMC-T and the ADK estimates are based on quasiclassical tunneling, the difference can be primarily attributed to the effect of laser envelope accounted for in the CTMC-T but not in the standard ADK approximation. As illustrated in Fig. 5, the width $\delta p$ calculated by the CTMC-T, $\delta p_{\text {СTMC }}$, depends on the pulse duration. As the pulse duration increases, $\delta p_{\text {СтMC }}$ becomes smaller overall and the slope $d\left(\delta p_{\text {СтMC }}\right) / d I_{\max }$ decreases as well. This tendency indicates a saturation of ionization, i.e., atoms never experience the peak intensity $I_{\max }$ since they tunnel and ionize before the laser reaches its peak $[28,35]$. In the limit of infinitely long pulse, ionization saturates when the temporal intensity of the laser is roughly given by the appearance intensity $I_{q}$ and therefore $\delta p_{\text {СтMC }}$ is determined by $I_{q}$ and independent of the peak intensity $I_{\max }$. With a finite duration of the laser, electrons do not have enough time to be ionized while the laser intensity is around $I_{q}$ and, therefore, ionization saturates at intensities slightly larger than $I_{q}$ leading to larger values of $\delta p_{\text {СТмС }}$. Since the larger peak intensity corresponds to a faster ramping of the laser amplitude, the saturation intensity and, correspondingly, the resulting momentum width $\delta p_{\text {СTMC }}$ increases as a function of the peak intensity (see Fig. 5). One should also realize the sensitivity of $\delta p$ on the interval used for the peak intensity averaging. Within the CTMC-T model we have simply restricted the intensity integration to the interval $I_{q}<I_{\max }<I_{q+1}$ deter- 
mined from a classical ionization threshold [Eq. (8)]. A more realistic choice of an integration range might influence the final width $\delta p$. The width $\delta p$ is, however, a slowly increasing function with a peak intensity, especially for a laser pulse with a long pulse duration. The slope becomes small for higher intensity where the main contribution to ionization is expected. Therefore a modification of the integration range is not expected to strongly affect the width plotted in Fig. 4.

\section{CONCLUSIONS}

We have investigated the momentum distributions of ions with different target atoms and charge states driven by a very intense $\left(50-70 \mathrm{PW} / \mathrm{cm}^{2}\right)$ and relatively long (200 fs) laser pulse. While the ionization mechanism involves many electrons and, in principle, is very complicated, the resulting width of momentum distributions appears to be surprisingly simple displaying an approximate power-law dependence on the ionization potential of ions with an exponent close to unity. This behavior is largely independent of the target atom. A simplified theoretical study using a single active electron model reproduces the observed nearly linear dependence on the ionization potential. In this laser parameter regime (intensity and long pulse duration), the observed behavior is dominated by the interaction of the active electrons with the laser field and electron-electron interactions are of minor importance. For a relatively long (200 fs) laser pulse, the effect of laser envelope becomes significant and plays an important role for the momentum width. It is interesting to study a similar ionization process with a shorter laser pulse where the effect of the laser envelope manifests itself differently and the electron-electron correlation should become more important.

\section{ACKNOWLEDGMENTS}

This research is supported by the FWF-SFB-F016 (Austria) and EU-HITRAP Project No. HPRI-CT-2001-50067. J.B. also acknowledges support from the RIKEN Eminent Scientist program.
[1] B. Walker, B. Sheehy, L. F. DiMauro, P. Agostini, K. J. Schafer, and K. C. Kulander, Phys. Rev. Lett. 73, 1227 (1994).

[2] A. Talebpour, C.-Y. Chien, Y. Liang, S. Larochelle, and S. L. Chin, J. Phys. B 30, 1721 (1997).

[3] S. Larochelle, A. Talebpour, and S. L. Chin, J. Phys. B 31, 1201 (1998).

[4] J. B. Watson, A. Sanpera, D. G. Lappas, P. L. Knight, and K. Burnett, Phys. Rev. Lett. 78, 1884 (1997).

[5] G. L. Yudin and M. Y. Ivanov, Phys. Rev. A 64, 013409 (2001).

[6] P. J. Ho and J. H. Eberly, Phys. Rev. Lett. 95, 193002 (2005).

[7] S. Palaniyappan, A. DiChiara, E. Chowdhury, A. Falkowski, G. Ongadi, E. L. Huskins, and B. C. Walker, Phys. Rev. Lett. 94, 243003 (2005).

[8] M. V. Ammosov, N. B. Delone, and V. P. Krainov, Sov. Phys. JETP 64, 1191 (1986).

[9] N. B. Delone and V. P. Krainov, J. Opt. Soc. Am. B 8, 1207 (1991).

[10] H. Wabnitz, A. R. B. de Castro, P. Gurtler, T. Laarmann, W. Laasch, J. Schulz, and T. Moller, Phys. Rev. Lett. 94, 023001 (2005).

[11] T. Weber et al., Phys. Rev. Lett. 84, 443 (2000a).

[12] R. Moshammer et al., Phys. Rev. Lett. 84, 447 (2000).

[13] T. Weber, H. Giessen, M. Weckenbrock, G. Urbasch, A. Staudte, L. Spielberger, O. Jagutzki, V. Mergel, M. Vollmer, and R. Dörner, Nature (London) 405, 658 (2000b).

[14] T. Weber et al., J. Phys. B 33, L127 (2000c).

[15] B. Feuerstein, R. Moshammer, and J. Ullrich, J. Phys. B 33, L823 (2000).

[16] R. Moshammer et al., Phys. Rev. Lett. 91, 113002 (2003).

[17] A. Rudenko, K. Zrost, C. D. Schröter, V. L. B. de Jesus, B. Feuerstein, R. Moshammer, and J. Ullrich, J. Phys. B 37, L407 (2004a).

[18] D. Comtois, D. Zeidler, H. Pépin, J. C. Kieffer, D. M. Ville- neuve, and P. B. Corkum, J. Phys. B 38, 1923 (2005).

[19] P. J. Ho, R. Panfili, S. L. Haan, and J. H. Eberly, Phys. Rev. Lett. 94, 093002 (2005).

[20] J. Chen, J. Liu, L. B. Fu, and W. M. Zheng, Phys. Rev. A 63, 011404 (2000).

[21] A. Rudenko, K. Zrost, B. Feuerstein, V. L. B. de Jesus, C. D. Schroter, R. Moshammer, and J. Ullrich, Phys. Rev. Lett. 93, 253001 (2004b).

[22] H. Shimada, Y. Nakai, H. Oyama, K. Ando, T. Kambara, A. Hataleyama, and Y. Yamzaki, Nucl. Instrum. Methods Phys. Res. B 235, 221 (2005).

[23] J. S. Cohen, Phys. Rev. A 64, 043412 (2001).

[24] D. G. Arbó, S. Yoshida, E. Persson, K. I. Dimitriou, and J. Burgdörfer, Phys. Rev. Lett. 96, 143003 (2006).

[25] D. Strickland and G. Mourou, Opt. Commun. 56, 219 (1985).

[26] P. P. Szydlik and A. E. S. Green, Phys. Rev. A 9, 1885 (1974).

[27] R. H. Garvey, C. H. Jackman, and A. E. S. Green, Phys. Rev. A 12, 1144 (1975).

[28] S. M. Hankin, D. M. Villeneuve, P. B. Corkum, and D. M. Rayner, Phys. Rev. A 64, 013405 (2001).

[29] S. Augst, D. Strickland, D. D. Meyerhofer, S. L. Chin, and J. H. Eberly, Phys. Rev. Lett. 63, 2212 (1989).

[30] K. I. Dimitriou, D. G. Arbo, S. Yoshida, E. Persson, and J. Burgdörfer, Phys. Rev. A 70, 061401(R) (2004).

[31] C. O. Reinhold and C. A. Falcón, Phys. Rev. A 33, 3859 (1986).

[32] R. Abrines and I. C. Percival, Proc. Phys. Soc. London 88, 861 (1966).

[33] R. E. Olson, J. Ullrich, and H. Schmidt-Böcking, Phys. Rev. A 39, 5572 (1989).

[34] M. Wickenhauser, X. M. Tong, and C. D. Lin, Phys. Rev. A 73, 011401(R) (2006).

[35] P. Lambropoulos, Phys. Rev. Lett. 55, 2141 (1985). 